\title{
Knightian Uncertainty and Insurance Regulation Decision
}

\author{
An Chen * \\ $\mathrm{Xia} \mathrm{Su}^{\dagger}$ \\ July 29,2008
}

\begin{abstract}
In contrast to insurance companies, regulatory authorities or regulators can obtain only limited information about the companies' value. It hence leads to some effects on the regulation design, which is however often overlooked in the literature. This paper characterizes the limited/imperfect information as Knightian [1921] uncertainty (ambiguity). In order to stress the analytical effects of ambiguity on the regulation decisions, we firstly carry out an analysis in a standard immediate bankruptcy regulation where default and liquidation are considered as indistinguishable events. It is noticed that ambiguityaverse regulators require more "ambiguity equity". We show then that under ambiguity an immediate liquidation policy delivers false liquidation with a positive probability. As an illustrative example to fix the false liquidation problem under ambiguity, a new regulation rule is developed with a regulatory auditing process. Based on this new model setup, we focus on examining how the riskiness of the firm's value and the debt ratio affect liquidation probability.
\end{abstract}

Keywords: Ambiguity, ambiguity equity, optimal regulation, default and liquidation design

Journal of Economic Literature Classification: D80, G22, G33

MathSci Classification Codes : 62P05, 91B28

\footnotetext{
*(Corresponding author) Netspar and Department of Quantitative Economics, Faculty of Economics and Business, University of Amsterdam, Roetersstraat 11, 1018 WB Amsterdam, The Netherlands, Phone: +31-20-5254125, Fax: +31-20-5254349, Email: A.Chen@uva.nl.

${ }^{\dagger}$ Bonn Graduate School of Economics, University of Bonn, Adenauerallee 24-42, 53113 Bonn, Germany.

The authors thank Michael Suchanecki and Stefan Trautmann for their helpful comments.
} 


\section{Introduction}

Solvency requirement becomes increasingly important with the insurance undertaking of Solvency II which aims to provide a guide to the new framework for prudential regulation in the EU. Together with the current trend of studying how to develop new regulatory methods and tools in order to reduce the insolvency and to better the benefit of the policy holders, it implies that the role of the regulator has been and will be more strengthened. In fact, the current regulation is still to be strongly reinforced because the collapse of many insurance companies is closely related to the insufficient regulation. For instance, the fall of First Executive Life Insurance Co. provides important lessons in regulation of life insurance companies ${ }^{1}$ Conceiving this purpose, this paper investigates the insurance regulation design with a special focus on the information transparency of insurance undertakings in the regulation decision.

In the standard insurance regulation literature, it is often supposed that regulators have full information on the insurer's asset value. The nullification of this assumption can be however justified by the fact that insurance companies have some firm-specific information and regulators are able to use only information external to the firm, for instance, some industry-wise measures. Indeed, the classical model rules out the situation where regulators are not sure of the likelihood of states of the world, i.e., the future asset evolution of each single insurance company. In other words, it adopts a strict assumption about regulators' belief: they are perfectly certain of the objective probability law of the state process and this belief is perfectly identical to the true probability law. However, regulators, as outsiders, may not have perfect confidence on the perceived probability measure for insurance companies' future asset value. With imprecise information, he may claim the possibility of other probability measures and has no idea of the true plausibility of those measures. This is exactly the ambiguity defined by Knight [1921], which refers to the uncertainty to the underlying probabilities. Through experiments and theoretical proofs, it has been long demonstrated that such uncertainty is not reducible to a single probability and has a great effect on decisions. Our work hence focuses on the impact of ambiguity on the insurance regulations and on the design of proper regulation rules under ambiguity. To our knowledge, it is the first paper involving ambiguity feature in the insurance modelling framework.

This paper first aims at showing the impact of the ambiguity on the regulation decisions. The analysis is conducted in a simplified framework as proposed in the literature (c.f. for instance Grosen and Jørgensen [2002]) to model default and bankruptcy, i.e., an standard bankruptcy regulation which does not distinguish between default and bankruptcy. We mainly examine how regulators establish an optimal regulation (barrier) level as an

\footnotetext{
${ }^{1}$ C.f. Schulte [1991] provides an insider's view on the the fall of First Executive Life Insurance Co.
} 
intervention under ambiguity. The barrier level is chosen such that the default probability is restricted to some fixed limit, say, to have a default probability not higher than $1 \%$. We show then that risk-neutral but ambiguity-averse regulators determine the optimal barrier level by choosing the worst scenario in all possible probability measures. Both analytical and numerical results demonstrate that ambiguity obviously affects the default probability and hence the regulation parameter determination. Especially, an ambiguity-averse regulator will force the regulated firm to carry more equity in order to meet the default probability constraint. This requirement in higher equity is not due to the higher riskiness of the capital but due to the existence of ambiguity. In this sense, it is defined as "ambiguity equity". All these results imply that ambiguity issue should be taken into consideration when designing insurance regulation.

As the second contribution of this paper, we show that an immediate default regulation brings false liquidation under ambiguity with a positive probability and design a new default and liquidation model under ambiguity in order to better fit the ambiguity framework. The conventional default modelling framework has an obvious drawback under ambiguity. Bankruptcy may be declared by a regulator with beliefs $Q^{\theta}$ (different from the real world measure $P$ ), even when the firm value is still sufficiently high (under $P$ ). Such situations cannot be avoided when default and liquidation are put into action simultaneously. Another regulation rule is hence required to well incorporate ambiguity. Instead of immediate liquidation, our default and liquidation formulation contains a regulatory auditing rules and hence prevents the mistaken liquidation resulted from ambiguity. In principle, firms can only be liquidated when the firm's asset value falls below the barrier level (under $P$ ). We then focus on the recognition and correction ability of the newly developed regulation policy under ambiguity by analyzing the impact of debt ratio and the riskiness of the firm's asset on liquidation probability.

The model we develop in this paper follows two streams of literature. On the one hand, it is related to the insurance default and regulation literature. In the existing literature of default modeling, Briys and de Varenne [1994, 1997, 2001] first interpret the work of Merton [1974, 1989] in an insurance context, in which only default at maturity is possible. Grosen and Jørgensen [2000, 2002] extends the model by allowing possible default at any instant before maturity. Bernard, Le Courtois and Quittard-Pinon [2005, 2006] and Chen and Suchanecki [2007] further extend Grosen and Jørgensen by either incorporating stochastic interest rate or discussing more realistic bankruptcy procedures. In all the literature, the regulators act very passively in the sense of not taking any actions against the collapse of the insurance company when it runs into default. This is apparently counterfactual. Bernard and Chen [2008] carry out an analysis from a regulator's viewpoint, investigating how regulators can establish regulatory rules to meet some regulatory ob- 
jectives and finally to improve the benefits of the policyholders. In the first part of this paper, we incorporate ambiguity and make a similar analysis as in Bernard and Chen [2008].

On the other hand, our work is related to the work of decision making under ambiguity. The Ellsberg [1961] paradox first demonstrates that risk and uncertainty are (behaviorally) meaningfully distinct from each other. The ambiguity theory is further prominently developed by Schmeidler [1989] (the Expected Utility Theory) and Gilboa and Schmeidler [1989] (the Multiple Expected Utility theory). The present paper carries on the formulation of the second theory, using the continuous-time implementation by Chen and Epstein [2002]. The incompleteness caused ambiguity is also applied by Nishimura and Ozaki [2004] in irreversible investment.

The remainder of this paper is organized in the following way: Section 2 gives the model framework and introduces Chen and Epstein's [2002] model of ambiguity. The impact of ambiguity on the determination of the regulation rules is demonstrated in Section 3 by using the standard but simplified model of immediate bankruptcy regulation. Section 4 poses the problem of the conventional immediate bankruptcy policy under ambiguity. In order to avoid the possible false liquidation due to ambiguity, a new audit regulatory rule is then illustrated. Numerical results are provided on how the new regulation corrects ambiguity. The result should be of interest to regulators as well as insurers. Finally, Section 5 concludes the paper with a short summary of the results and an outlook on the future research.

\section{Model Setup}

In this paper, we examine the regulation of an insurance company that offers only one type of policy. These contacts grant policyholders a minimum interest rate guarantee and a participation clause in the surpluses of the company's value, if any. Such a product is fairly popular and can be a with-profit contract as introduced in Bacinello [2001] or Ballotta [2005] among others, a French participating contract in Briys and de Varenne [1994] as well as an equity-linked product etc.. In addition to the standard model construction, we involve ambiguity in the model and show its effects on the insurance regulation.

\subsection{Adopted Framework}

The standard assumptions of the Black and Scholes [1973] framework are made in this paper. There exists a risk free asset which is continuously compounded with a constant instantaneous interest rate $r$. Trading takes place continuously and there are no other transaction costs, tax and so on. 
Guarantee payment A payment is provided to the policyholder at the maturity date $T$, when there is no premature default. The payoff of the contract can be usually decomposed into three parts:

$$
\text { Guarantee + Call - Put, }
$$

namely, a guaranteed amount, a long position in a call option which is in effect a bonus option given to policyholders as a participation in the performance of the firm's assets and a short position in a put (the default put). Particularly, it is assumed that a guarantee is provided at the maturity date of the contract, $T$ :

$$
G_{T}=G_{0} e^{g T}
$$

The guaranteed amount corresponds to an initial investment $G_{0}$ accumulated with a minimum continuously-compounded interest rate $g$. In order to protect insurers, regulators usually establish certain regulatory rules. The whole effect of such regulations is absorbed in the guarantee part; while the other two parts of call and put are designed by each single insurance company as the highlights of the contract. Therefore, we are concerned in the following analysis only with the guarantee part.

Premature default formulation A premature default is assumed to result in an immediate liquidation. A premature liquidation occurs when the firm's assets hit the barrier level (which is set by the external regulators) before the maturity date and is constructed as a first passage time problem. Generally, the critical barrier level $B_{t}$ is set up by regulators. Since the firm's assets $A_{t}$ should be high enough to meet the increasing guaranteed amount, it is not unreasonable to assume a time-increasing barrier:

$$
B_{t}(\eta, \phi)=\eta G_{0} e^{\phi t}, \eta<\frac{A_{0}}{G_{0}}
$$

for $t \in[0, T] . \eta<\frac{A_{0}}{G_{0}}$ ensures that the insurance company is solvent at the contract-issuing time. It should be pointed out that the barrier function $B_{t}(\eta, \phi)$ can exhibit quite flexible patterns. It is observed that this time-varying barrier encompasses the exponential barrier given in Grosen and Jørgensen [2002], Chen and Suchanecki [2007] and Bernard and Chen [2008] by setting $\phi=g$. Here, both $\eta$ and $\phi$ can be used by the regulator to control the strictness of the auditing rule. A high $\eta$ or a high $\phi$ would lead to a high barrier level. Because the default time is associated with the ambiguity specification, we postpone introducing its mathematical formulation in a later section. 


\subsection{Ambiguity: Uncertainty in the Underlying Probability Mea- sures}

Uncertainty and risk are usually not distinguished in the financial and actuarial literature. However, according to Knight [1921], risk is the so-called "uncertainty" which can be reduced to a single probability; while the true uncertainty is not reducible. In the famous urn experiment ${ }^{2}$, Ellsberg [1961] demonstrated that uncertainty, different from risk, has an impact on decisions: one prefers known to unknown or ambiguous probabilities. Besides in experimental settings, there are many obvious instances in our real life. For example, ambiguity is addressed to explain the equity premium puzzle whereby the representative agent model fails to fit historical averages of the equity premium and the risk-free interest rate. With uncertainty in the underlying probabilities, it is argued that part of the premium is indeed due to the greater ambiguity associated with the return to equity, which hence reduces the required degree of risk aversion. Meanwhile, Ellsberg's findings suggest that decision makers tend to be uncertainty or ambiguity averse.

Although risk and uncertainty are clearly distinct concepts, they are little treated separately in finance and almost never in insurance. Indeed, even if the firm has perfect information on his own firm value's future evolution, regulators and the insured do not. This fact is utmost important in many aspects. Typically, regulators set up a certain regulation with limited information. Most probably, they have only industry-specific probability measure and are not confident about the assets evolution of each single firm. This is exactly the ambiguity defined by Knight. In turn, it exerts a great impact on the insurance regulations. In the following, we formulate the ambiguity in such a way that the regulator is not sure that a given (i.e. predicted for the entire industry) probability measure is the true one. The regulators' beliefs are constructed by a set of probability measures instead of a single probability measure.

\subsubsection{Ambiguity in Continuous Time}

Assume that the insurance company has some firm-specific information and the regulator can only use information external to the firm, for instance some industry-wide measures as well as publicly available financial information of the insurer such as the insurer's financial reports or its share prices, if the insurer has demutualized. Therefore, the regulator has

\footnotetext{
${ }^{2}$ To roughly outline the Ellberg paradox, suppose that we have two urns. There are exactly 10 red and 10 blue balls in the first urn. The second one contains also altogether 20 balls either in red or in blue. However, there is no clear number of red or blue balls. The experiment is designed to check the willingness of persons who are offered a bet on drawing a red ball from the two urns. The result shows that a majority of persons choose to draw from the first urn rather than the second. The result remains when drawing a blue ball.
} 
uncertainty about the future evolution of the insurer's assets and it is assumed that this uncertainty is captured by ambiguity. In case of ambiguity, we make a crucial assumption that the regulator can only observe the insurer's initial firm value $A_{0}$ and terminal value $A_{T}$. While $A_{t}, t \in(0, T)$, is not directly observable to the regulator unless a regulatory auditing process is initiated against the insurer. More specifically, the regulator's ambiguity about the firm's assets $A_{t}$ is characterized by a set of priors $\mathcal{P}^{\Theta}$ which are equivalent to the real world measure $P$. The set of the priors can be determined by constructing a set of suitable densities which is further specified by a series of density generators $\Theta$. Each density generator $\left(\theta_{t}\right) \in \Theta$ is assumed to be an $\mathbb{R}$-valued process for which the process $\left(z_{t}^{\theta}\right)$ is a $P$-martingale, i.e.

$$
d z_{t}^{\theta}=-z_{t}^{\theta} \theta_{t} d W_{t}, \quad z_{0}^{\theta}=1
$$

where $W_{t}$ is a Brownian motion under $P$ and $\theta$ satisfies the Novikov condition:

$$
E\left[\exp \left\{\frac{1}{2} \int_{0}^{T}|\theta|^{2} d s\right\}\right]<\infty
$$

Solving this stochastic equation leads to

$$
z_{t}^{\theta}=\exp \left\{-\frac{1}{2} \int_{0}^{t}\left|\theta_{s}\right|^{2} d s-\int_{0}^{t} \theta_{s} \cdot d W_{s}\right\}, \quad 0 \leq t \leq T .
$$

Given the set $\Theta$ of density generators, the corresponding set of priors is

$$
\mathcal{P}^{\Theta}=\left\{Q^{\theta}: \theta \in \Theta \text { and } Q^{\theta} \text { is defined by } \frac{d Q^{\theta}}{d P}=z_{T}^{\theta}\right\},
$$

which is mutually absolutely continuous with respect to $P$. Let $\left(W_{t}^{\theta}\right)_{t \in[0, T]}$ denote the Brownian motion under $Q^{\theta}$, it holds $d W_{t}^{\theta}=d W_{t}+\theta_{t} d t$.

The above construction of ambiguity in continuous time follows basically the work of Chen and Epstein [2002]. In this setup, it is assumed that the firm considers only a set of probability measures that have perfect agreement with $P$ with respect to zero probability events. It implies that all probability measures considered in the paper are absolutely continuous with respect to $P$ and one another, as given above.

Under the real world probability measure $P$, the firm's value evolves according to the following stochastic backward differential equation:

$$
d A_{t}=\mu A_{t} d t+\sigma A_{t} d W_{t}
$$

where $\mu$ and $\sigma>0$ are instantaneous rate of return and volatility of the asset. Rather than the single probability measure $P$, we now generalize the stochastic differential equation 
by allowing the regulator to employ the set $\mathcal{P}^{\Theta}$. For a given $Q^{\theta} \in \mathcal{P}^{\Theta}$ which describes a regulator with probability belief $Q^{\theta}$, the firm's assets evolve as

$$
d A_{t}^{\theta}=\left(\mu+\sigma \theta_{t}\right) A_{t}^{\theta} d t+\sigma A_{t}^{\theta} d W_{t}
$$

In order to account for the fact $W_{t}$ is not a Brownian motion under $Q^{\theta}$, the drift is adjusted by the addition $\sigma \theta_{t}$ compared to Equation (3).

We assume that the initial value $A_{0}^{\theta}=A_{0}$ takes the same value for all $\theta$. Clearly, those stochastic processes in the set $\mathcal{P}^{\Theta}$ vary only in the drift term. The uncertainty/ambiguity in the probability measures gives rise to some different stochastic processes to specify or interpret. Moreover, their confidence over probability measures is supposed to be not (greatly) improved over time $3^{3}$ Formally, it is not possible to learn the distribution of $\theta \in \Theta$, neither to reduce the set $\mathcal{P}^{\Theta}$ over time.

As assumed in the literature, all the candidate probability measures are naturally not widely from the industry-specific measure, but with a small deviation. In particular, the density generators $\left(\theta_{t}\right) \in \Theta$ is supposed to be restricted to the range $[-\kappa, \kappa]$. It is the so-called $\kappa$-ignorance (Chen and Epstein [2002]) and $\kappa$ can be considered as the degree of Knightian uncertainty or ignorance. It is clear that there is no uncertainty if $\kappa=0$. When $\kappa$ increases, it implies that regulators are less certain about how good the public information (industry level) represents the individual firm's performance. ${ }^{4}$

\section{Optimal Standard Immediate Bankruptcy Rule un- der Ambiguity}

This section aims at investigating the impact of ambiguity on the regulation decisions analytically. To this end, the standard immediate bankruptcy rule is considered. Under the standard rule, the regulator declares liquidation of the firm immediately when the proxy $A_{t}$ breaches the predetermined default threshold. The premature default time or premature liquidation time is defined as

$$
\tau:=\inf \left\{t \leq T \mid A_{t} \leq B_{t}(\eta, \phi) .\right\}
$$

\footnotetext{
${ }^{3}$ It is also plausible when considering that regulations are made for some amount of time without great change.

${ }^{4}$ As shown in Chen and Epstein [2002], $\kappa$-ignorance is a special case of i.i.d uncertainty and has the property of strong rectangularity, which are two critical conditions required by the ambiguity theory. Especially, the strong regularity guarantees the optimality in a recursive structure, i.e., $\max _{\theta \in \Theta} E^{Q^{\theta}}\left[x \mid \mathcal{F}_{s}\right]=\max _{\theta \in \Theta} E^{Q^{\theta}}\left[E^{Q^{\theta}}\left[x \mid \mathcal{F}_{t}\right] \mid \mathcal{F}_{s}\right]=\max _{\theta \in \Theta} E^{Q^{\theta}}\left[\max _{\theta^{\prime} \in \Theta} E^{Q^{\theta^{\prime}}}\left[x \mid \mathcal{F}_{t}\right] \mid \mathcal{F}_{s}\right]$. The detailed technique is referred to Chen and Epstein [2002].
} 
$\tau$ is the first passage time that $A_{t}$ hits the barrier $B_{t}(\eta, \phi)$ from above. Similarly as in Bernard and Chen [2008], it is assumed that the regulator acts as "default-probability minimizer $5^{5}$. Formally, the regulator with $Q^{\theta}$-beliefs is concerned with the default probability

$$
Q^{\theta}(\tau \leq T)
$$

It shall be emphasized that a $Q^{\theta}$-regulator's regulation policy is based on the beliefconstructing probability measure $Q^{\theta}$ rather than the real world measure $P$.

Proposition 3.1 (Default Probability) A "default-probability minimizing" regulator with $Q^{\theta}$-beliefs, who is concerned with the default probability

$$
Q^{\theta}(\tau \leq T)
$$

under $\kappa$-ignorance, i.e., $\left(\theta_{t}\right) \in \Theta$ are restricted to the range $[-\kappa, \kappa]$, achieves the minimum default probability when the ambiguity parameter equals to the "lower-rim density generator" $-\kappa$

$$
\min _{\theta \in \Theta} Q^{\theta}(\tau \leq T)=Q^{-\kappa}(\tau \leq T)=N\left(d_{2}^{T}\left(\hat{\mu}^{*}\right)\right)+\left(\frac{A_{0}}{\eta G_{0}}\right)^{\frac{-2 \hat{\mu}^{*}}{\sigma^{2}}} N\left(d_{1}^{T}\left(\hat{\mu}^{*}\right)\right)
$$

with $\hat{\mu}^{*}=\mu+\sigma \kappa-\phi-\frac{\sigma^{2}}{2}$. The maximum default probability is obtained when $\theta=\kappa$ :

$$
\max _{\theta \in \Theta} Q^{\theta}(\tau \leq T)=Q^{\kappa}(\tau \leq T)=N\left(d_{2}^{T}\left(\hat{\mu}^{* *}\right)\right)+\left(\frac{A_{0}}{\eta G_{0}}\right)^{\frac{-2 \hat{\mu}^{* *}}{\sigma^{2}}} N\left(d_{1}^{T}\left(\hat{\mu}^{* *}\right)\right)
$$

with $\hat{\mu}^{* *}=\mu-\sigma \kappa-\phi-\frac{\sigma^{2}}{2}$.

Proof: According to the firm's assets evolution in Equations (3), it is possible to calculate this cumulative default probability in closed form (c.f. e.g. Karatzas and Shreve [1991]):

$$
\begin{aligned}
Q^{\theta}(\tau \leq T) & =Q^{\theta}\left(\inf _{t \in[0, T]} \hat{\mu} t+\sigma W_{t}^{\theta}<D_{0}\right)=N\left(d_{2}^{T}(\hat{\mu})\right)+\left(\frac{A_{0}}{\eta G_{0}}\right)^{\frac{-2 \hat{\mu}}{\sigma^{2}}} N\left(d_{1}^{T}(\hat{\mu})\right) \\
d_{2}^{T}(\hat{\mu}) & =\frac{\ln \left(\frac{\eta G_{0}}{A_{0}}\right)-\hat{\mu} T}{\sigma \sqrt{T}} \\
d_{1}^{T}(\hat{\mu}) & =\frac{\ln \left(\frac{\eta G_{0}}{A_{0}}\right)+\hat{\mu} T}{\sigma \sqrt{T}}
\end{aligned}
$$

with $\hat{\mu}=\mu-\sigma \theta-\phi-\frac{\sigma^{2}}{2}$ and $D_{0}=\frac{1}{\sigma} \ln \frac{\eta G_{0}}{A_{0}}$. In the first step we have used the substitution $W_{t}=W_{t}^{\theta}-\theta t$.

\footnotetext{
${ }^{5}$ Solvency II advocates a risk-based regulation which focuses on downside risk, i.e. it is likely to require insurance companies to use measures such as probability of ruin, value-at-risk or tail value-at-risk. In our context, the regulator tries to control the ruin probability by establishing regulation rules.
} 
It is trivial that $\theta$ has the reversed effect on the cumulative default probability as $\hat{\mu}$. This simplifies the problem of how the ambiguity parameter influences the default probability by checking the effect of $\hat{\mu}$ on the probability. To this end, the derivative of the default probability with respect to $\hat{\mu}$ is calculated

$$
\begin{aligned}
\frac{\partial Q^{\theta}(\tau \leq T)}{\partial \hat{\mu}} & =-\frac{\sqrt{T}}{\sigma} n\left(d_{2}^{T}(\hat{\mu})\right)-\frac{2}{\sigma^{2}} \ln \frac{A_{0}}{\eta G_{0}}\left(\frac{A_{0}}{\eta G_{0}}\right)^{\frac{-2 \hat{\mu}}{\sigma^{2}}} N\left(d_{1}^{T}(\hat{\mu})\right)+\frac{\sqrt{T}}{\sigma}\left(\frac{A_{0}}{\eta G_{0}}\right)^{\frac{-2 \hat{\mu}}{\sigma^{2}}} n\left(d_{1}^{T}(\hat{\mu})\right) \\
& =-\frac{2}{\sigma^{2}} \ln \frac{A_{0}}{\eta G_{0}}\left(\frac{A_{0}}{\eta G_{0}}\right)^{\frac{-2 \hat{\mu}}{\sigma^{2}}} N\left(d_{1}^{T}(\hat{\mu})\right)<0
\end{aligned}
$$

where $n(x)=\frac{1}{\sqrt{2 \pi}} e^{-\frac{x^{2}}{2}}$ denotes the density of a standard normally distributed random variable $x$ and the second step is shown by the following argument:

$$
\begin{aligned}
& -\frac{\sqrt{T}}{\sigma} n\left(d_{2}^{T}(\hat{\mu})\right)+\frac{\sqrt{T}}{\sigma}\left(\frac{A_{0}}{\eta G_{0}}\right)^{\frac{-2 \hat{\mu}}{\sigma^{2}}} n\left(d_{1}^{T}(\hat{\mu})\right) \\
= & -\frac{\sqrt{T}}{\sigma} \frac{1}{\sqrt{2 \pi}} e^{-\frac{\left(d_{2}^{T}\right)^{2}}{2}}+\frac{\sqrt{T}}{\sigma} \frac{1}{\sqrt{2 \pi}} e^{-\frac{\left(d_{2}^{T}\right)^{2}}{2}}\left(\frac{A_{0}}{\eta G_{0}}\right)^{\frac{-2 \hat{\mu}}{\sigma^{2}}} \exp \left\{-\frac{2 d_{2}^{T} \hat{\mu} \sqrt{T}}{\sigma}\right\} \exp \left\{-\frac{2 \hat{\mu}^{2} T}{\sigma^{2}}\right\} \\
= & -\frac{\sqrt{T}}{\sigma} \frac{1}{\sqrt{2 \pi}} e^{-\frac{\left(d_{2}^{T}\right)^{2}}{2}}+\frac{\sqrt{T}}{\sigma} \frac{1}{\sqrt{2 \pi}} e^{-\frac{\left(d_{2}^{T}\right)^{2}}{2}}\left(\frac{A_{0}}{\eta G_{0}}\right)^{\frac{-2 \hat{\mu}}{\sigma^{2}}}\left(\frac{\eta G_{0}}{A_{0}}\right)^{\frac{-2 \hat{\mu}}{\sigma^{2}}} \exp \left\{\frac{2 \hat{\mu}^{2} T}{\sigma^{2}}\right\} \exp \left\{-\frac{2 \hat{\mu}^{2} T}{\sigma^{2}}\right\} \\
= & 0 .
\end{aligned}
$$

The negative relation between the default probability and $\hat{\mu}$ indicates that the default probability goes up with the ambiguity parameter $\theta$. Therefore, it holds

$$
\begin{gathered}
\min _{\theta \in \Theta} Q^{\theta}(\tau \leq T)=Q^{-\kappa}(\tau \leq T) . \\
\max _{\theta \in \Theta} Q^{\theta}(\tau \leq T)=Q^{\kappa}(\tau \leq T) .
\end{gathered}
$$

In other words, under $\kappa$-ignorance, i.e. $\left(\theta_{t}\right) \in \Theta$ are restricted to the range $[-\kappa, \kappa]$, the minimum default probability is achieved when the ambiguity parameter equals to the "lower-rim density generator" $\kappa$ and is given by

$$
\min _{\theta \in \Theta} Q^{\theta}(\tau \leq T)=N\left(d_{2}^{T}\left(\hat{\mu}^{*}\right)\right)+\left(\frac{A_{0}}{\eta G_{0}}\right)^{\frac{-2 \hat{\mu}^{*}}{\sigma^{2}}} N\left(d_{1}^{T}\left(\hat{\mu}^{*}\right)\right)
$$

with $\hat{\mu}^{*}=\mu+\sigma \kappa-\phi-\frac{\sigma^{2}}{2}$ and the maximum default probability is obtained when $\theta=\kappa$ :

$$
\max _{\theta \in \Theta} Q^{\theta}(\tau \leq T)=N\left(d_{2}^{T}\left(\hat{\mu}^{* *}\right)\right)+\left(\frac{A_{0}}{\eta G_{0}}\right)^{\frac{-2 \hat{\mu}^{* *}}{\sigma^{2}}} N\left(d_{1}^{T}\left(\hat{\mu}^{* *}\right)\right)
$$

with $\hat{\mu}^{* *}=\mu-\sigma \kappa-\phi-\frac{\sigma^{2}}{2}$. 
The regulator will establish the regulation level, or more specifically the barrier level, in order to meet the solvency requirement. In contrast to Bernard and Chen [2008], the regulator owns now two regulation parameters $\eta$ and $\phi$. Trivially, the default probability decreases with both $\eta$ and $\phi$. For a given $\phi$, the lower $\eta$ is, the lower the barrier level is, and the less likely the firm's asset will hit the barrier. For a given $\eta$, the same relation holds for $\phi$ and the default probability. As $\eta$ approaches 0 , the default probability goes asymptotically to zero, i.e.,

$$
\lim _{\eta \rightarrow 0} Q^{\theta}(\tau \leq T) \rightarrow 0 .
$$

Moreover, any positive $\eta$-value would lead to a positive default probability. As $\phi$ approaches zero, the barrier level becomes a constant equal to $\eta G_{0}$. Thus, we assume that the regulator aims at achieving a default probability constraint $\varepsilon$, which in turn helps us (numerically) determine the optimal regulation level $\eta$ or $\phi$. As shown in the literature, a decision maker with ambiguity-aversion behaves according to the worst scenario rule. This basic rule is also examined in our context.

Proposition 3.2 (Ambiguity Aversion) An ambiguity-averse regulator behaves according to the worst scenario rule. Namely, he chooses the optimal regulation level which makes the worst scenario - maximal default probability- below the default probability constraint, i.e.,

$$
\max _{\eta \operatorname{or}} \max _{\theta \in \Theta} Q^{\theta}(\tau \leq T) \leq \varepsilon
$$

Proof: The inner max problem of the objective function is already solved in the previous proposition. Given the maximum value at $\theta=\kappa$, the default probability increaes with the two regulation levels $\eta$ and $\phi$. By increasing their values, the default probability rises. Thus, they can be numerically determined given the maximal default probability $\varepsilon$.

Since the emphasis of this part is laid on how ambiguity affects the insurance regulation, we do not restrict ourself to ambiguity-averse regulators. We allow "ambiguity-friendly" regulators, i.e. we are interested in formulating in what range of the optimal regulation level falls when an ambiguity regulator with $\kappa$ degree of ignorance is considered. As shown above, the ambiguity-averse regulator behave according to the worst scenario rule. In contrast, an ambiguity-friendly regulator is taking $\kappa$ degree of ignorance and chooses an optimal regulation level to make the most optimistic scenario - minimal default probability - binding, i.e.

$$
\max _{\eta \text { or }} \min _{\theta \in \Theta} Q^{\theta}(\tau \leq T)=\varepsilon
$$

Table 1 and 2 provide some optimal $\eta$ and $\phi$ values, respectively. $\eta^{*}\left(\phi^{*}\right), \eta_{\kappa}^{*}\left(\phi_{\kappa}^{*}\right)$ and $\eta_{-\kappa}^{*}\left(\phi_{-\kappa}^{*}\right)$ denote the optimal $\eta(\phi)$ obtained for regulators with ambiguity-neutral, averse and friendly regulators. First, a positive relation between $\eta$ and the default probability constraint $\varepsilon$ is observed, and also that between $\eta$ and $\phi$. Besides, $\eta(\phi)$ declines with the 


\begin{tabular}{|c|c|c|c|c|c|c|}
\hline \multirow{2}{*}{$\varepsilon$} & \multicolumn{3}{|c|}{$\sigma=0.10$} & \multicolumn{3}{c|}{$\sigma=0.15$} \\
\cline { 2 - 7 } & $\eta^{*}$ & $\eta_{\kappa}^{*}$ & $\eta_{-\kappa}^{*}$ & $\eta^{*}$ & $\eta_{\kappa}^{*}$ & $\eta_{-\kappa}^{*}$ \\
\hline \hline 0.01 & 0.73818 & 0.68413 & 0.79078 & 0.47162 & 0.43378 & 0.51114 \\
0.02 & 0.79008 & 0.73536 & 0.84197 & 0.52718 & 0.48629 & 0.56934 \\
0.03 & 0.82347 & 0.76879 & 0.87437 & 0.56479 & 0.52211 & 0.60839 \\
0.04 & 0.84867 & 0.79431 & 0.89853 & 0.59424 & 0.55032 & 0.63875 \\
0.05 & 0.86915 & 0.81524 & 0.91799 & 0.61888 & 0.57405 & 0.66400 \\
0.06 & 0.88653 & 0.83314 & 0.93436 & 0.64031 & 0.59478 & 0.68583 \\
0.07 & 0.90169 & 0.84887 & 0.94855 & 0.65942 & 0.61335 & 0.70521 \\
0.08 & 0.91519 & 0.86297 & 0.96110 & 0.67676 & 0.63027 & 0.72271 \\
0.09 & 0.92738 & 0.87578 & 0.97238 & 0.69271 & 0.64589 & 0.73873 \\
0.10 & 0.93853 & 0.88756 & 0.98263 & 0.70752 & 0.66045 & 0.75355 \\
\hline \hline
\end{tabular}

Table 1: Optimal $\eta$ levels with parameters: $A_{0}=100 ; G_{0}=80 ; T=10 ; \mu=0.06 ; g=0.02 ; \kappa=0.01 / \sigma ; \phi=0.02$.

volatility $\sigma$. A higher volatility leads to a higher default probability, as a compensation, either $\eta$ or $\phi$ shall be set lower to meet the same default constraint $\varepsilon$. More importantly, regulators' attitude towards ambiguity delivers an obvious effect on the optimal $\eta$ and hence the barrier level. Moving from the extreme case of $\theta=\kappa$ (ambiguity aversity) to $\theta=-\kappa$ (ambiguity-friendliness) results in an increase in the optimal $\eta$ value by over $10 \%$ and a quite big difference in the barrier level between 6.4 and 8. Comparing $\eta_{\kappa}^{*}$ and $\eta_{\kappa}^{*}$ with $\eta^{*}$, if the regulator uses $\eta_{\kappa}^{*}$ to determine the optimal barrier level, under $P$ the real firm's value falls below the barrier indeed with a smaller probability than $\varepsilon$. On the contrary, under $P$ the real firm's value falls below the barrier with a larger probability than $\varepsilon$, while using $\eta_{-\kappa}^{*}$.

Clearly, an ambiguity-averse regulator requires a much lower regulation level (and hence the barrier level) to achieve the same default probability. If an optimal regulation level $\eta^{*}$ (optimal for an ambiguity-neutral regulator) is applied to an regulator who is indeed ambiguity-averse, a higher default probability (than the default probability constraint) results. In other words, an ambiguity-averse regulator will ask for some "ambiguity premium" to achieve the same default probability. This term is first invented by Chen and Epstein [2002] in the context of equity premium puzzle. Instead of the usually-recognized premium for the riskiness of equity, it is the premium due to greater ambiguity associated with the return to equity. Similarly, some additional premium is required in this paper by ambiguity. More specifically, it can be a requirement in the capital structure of insurance companies. Obviously, by increasing the investment of the equity holder, the default probability is to some extent reduced. We thus call this additional equity requirement "ambiguity equity". 


\begin{tabular}{|c|c|c|c|c|c|c|}
\hline \multirow{2}{*}{$\varepsilon$} & \multicolumn{3}{|c|}{$\sigma=0.10$} & \multicolumn{3}{c|}{$\sigma=0.15$} \\
\cline { 2 - 7 } & $\phi^{*}$ & $\phi_{\kappa}^{*}$ & $\phi_{-\kappa}^{*}$ & $\phi^{*}$ & $\phi_{\kappa}^{*}$ & $\phi_{-\kappa}^{*}$ \\
\hline \hline 0.01 & 0.06625 & 0.05625 & 0.07625 & 0.01278 & 0.00278 & 0.02278 \\
0.02 & 0.07509 & 0.06509 & 0.08509 & 0.02660 & 0.01660 & 0.03660 \\
0.03 & 0.08068 & 0.07068 & 0.09068 & 0.03532 & 0.02532 & 0.04532 \\
0.04 & 0.08489 & 0.07489 & 0.09489 & 0.04185 & 0.03185 & 0.05185 \\
0.05 & 0.08831 & 0.07831 & 0.09831 & 0.04715 & 0.03715 & 0.05715 \\
0.06 & 0.09122 & 0.08122 & 0.10122 & 0.05165 & 0.04165 & 0.06165 \\
0.07 & 0.09376 & 0.08376 & 0.10377 & 0.05559 & 0.04559 & 0.06559 \\
0.08 & 0.09605 & 0.08605 & 0.10605 & 0.05911 & 0.04911 & 0.06911 \\
0.09 & 0.09812 & 0.08812 & 0.10812 & 0.06231 & 0.05231 & 0.07231 \\
0.10 & 0.10003 & 0.09003 & 0.11003 & 0.06525 & 0.05525 & 0.07525 \\
\hline \hline
\end{tabular}

Table 2: Optimal $\phi$ levels with parameters: $A_{0}=100 ; G_{0}=80 ; T=10 ; \mu=0.06 ; \kappa=0.01 / \sigma ; \eta=0.5$.

Table 3 illustrates the ambiguity equity as a function of the default probability constraint $\varepsilon$ and of ignorance degree $\kappa$ for three volatility levels. The higher the allowed default probability level $\varepsilon$, the more tolerant the regulator, the less ambiguity equity an ambiguity-averse regulator requires. Furthermore, the ambiguity equity increases in the degree of ignorance. That is, the more ambiguity averse the regulator is, the more ambiguity equity results. Please note that the ambiguity equity is indeed quite high overall. For instance, for a $\kappa=0.7(\sigma=0.2)$, the required equity shall be increased by 8.55075 , which corresponds to an increase in equity by $42.75 \%$. The ambiguity equity is then observed to rise with the volatility of the firm value.

Ambiguity is usually ignored in the literature and regulators considered in the most models are assumed to be ambiguity-neutral. The designed default probability $\varepsilon$ is then not able to be achieved in reality where incomplete information or ambiguity is observed and especially when the regulator is ambiguity-averse. Ambiguity-averse regulators behave themselves more "conservatively" and admit the "worst-scenario" rule, which corresponds to the case $\theta=\kappa$ in this context where a maximum default probability results and the optimal regulation level turns out to be lower than the ambiguity-neutral case. As a result, a special attention is called for by both theory and practice to ambiguity issue in the regulation design and optimal barrier level determination. 


\begin{tabular}{|c||c||c|c||}
\hline \multicolumn{1}{|c||}{$\varepsilon$} & \multicolumn{3}{c||}{ Ambiguity equity } \\
\cline { 2 - 4 } & $\sigma=0.10$ & $\sigma=0.15$ & $\sigma=0.20$ \\
\hline \hline 0.01 & 5.85795 & 6.41898 & 6.65300 \\
0.02 & 5.54101 & 6.20525 & 6.48772 \\
0.03 & 5.31166 & 6.04629 & 6.36416 \\
0.04 & 5.12418 & 5.9132 & 6.26013 \\
0.05 & 4.96221 & 5.7957 & 6.16774 \\
0.06 & 4.81775 & 5.68878 & 6.08317 \\
0.07 & 4.68623 & 5.58958 & 6.00424 \\
0.08 & 4.56474 & 5.49628 & 5.92958 \\
0.09 & 4.45132 & 5.40768 & 5.85825 \\
0.10 & 4.34457 & 5.3229 & 5.78959 \\
\hline \hline
\end{tabular}

\begin{tabular}{||c||c||c||c||}
\multirow{2}{*}{$\kappa$} & \multicolumn{3}{c||}{ Ambiguity equity } \\
\cline { 2 - 4 } & $\sigma=0.10$ & $\sigma=0.15$ & $\sigma=0.20$ \\
\hline \hline $0.005 / \sigma$ & 2.43446 & 2.90925 & 3.12005 \\
$0.006 / \sigma$ & 2.93338 & 3.48877 & 3.73554 \\
$0.007 / \sigma$ & 3.43583 & 4.06728 & 4.34808 \\
$0.008 / \sigma$ & 3.9416 & 4.64467 & 4.95766 \\
$0.009 / \sigma$ & 4.45046 & 5.22084 & 5.56422 \\
$0.010 / \sigma$ & 4.96221 & 5.7957 & 6.16774 \\
$0.011 / \sigma$ & 5.47662 & 6.36916 & 6.76818 \\
$0.012 / \sigma$ & 5.99347 & 6.94113 & 7.36552 \\
$0.013 / \sigma$ & 6.51256 & 7.51152 & 7.95972 \\
$0.014 / \sigma$ & 7.03365 & 8.08025 & 8.55075 \\
\hline \hline
\end{tabular}

Table 3: Ambiguity equity with parameters: $A_{0}=100 ; G_{0}=80 ; T=10 ; \mu=0.06 ; g=$ $0.02 ; \kappa=0.01 / \sigma ; \phi=0.02, \varepsilon=0.05$ (right table).

\section{Regulatory Auditing Rules under Ambiguity}

As demonstrated in the previous section, ambiguity indeed has a great effect on the regulation design in the classical model setup where default and liquidation event are equivalent. More importantly, it is easily observed from the above derivation that the regulation determination heavily depends on the regulator's beliefs $Q^{\theta}$. Thus, under the standard immediate bankruptcy rule with ambiguity, the regulators with $Q^{\theta}$ might declare bankruptcy of the firm although the firm still performs very well under the real world measure $P$. Under $\kappa$-ignorance, i.e., $\theta \in[-\kappa, \kappa]$, the probability that the $Q^{\theta}$-regulator declares liquidation although the firm's asset does not hit the barrier under the real world measure is given in the following proposition.

Proposition 4.1 (False Bankruptcy Declaration) $A Q^{\theta}$-regulator falsely declares liquidation, although the firm's asset does not hit the barrier under $P$, with the probability of

$$
\begin{aligned}
P\left(\left\{\tau^{-\theta} \leq T \text { and } \tau>T\right\}\right)= & N\left(\frac{D_{0}-\hat{\mu} T}{\sigma \sqrt{T}}\right)+\left(\frac{A_{0}}{\eta G_{0}}\right)^{\frac{-2 \hat{\mu}}{\sigma^{2}}} N\left(\frac{D_{0}+\hat{\mu} T}{\sigma \sqrt{T}}\right) \\
& -\left(N\left(\frac{D_{0}-m T}{\sigma \sqrt{T}}\right)+\left(\frac{A_{0}}{\eta G_{0}}\right)^{\frac{-2 m}{\sigma^{2}}} N\left(\frac{D_{0}+m T}{\sigma \sqrt{T}}\right)\right)
\end{aligned}
$$

where $m=\mu-\phi-\frac{1}{2} \sigma^{2}$ and $D_{0}=\ln \frac{\eta G_{0}}{A_{0}}$.

Proof: Please note first that it holds $Q^{\theta}(\tau<T)=P\left(\tau^{-\theta}<T\right)$, where $\tau^{-\theta}$ is defined similarly as $\tau$ :

$$
\tau^{-\theta}:=\inf \left\{t \leq T \mid A_{t}^{-\theta} \leq B_{t}(\eta, \phi)\right\}
$$




\begin{tabular}{||c||cccccc||}
\hline \hline$\sigma$ & 0.10 & 0.12 & 0.14 & 0.16 & 0.18 & 0.20 \\
\hline \hline Probability & 0.012 & 0.0282 & 0.0443 & 0.0566 & 0.0645 & 0.0684 \\
\hline \hline
\end{tabular}

Table 4: Probability of Type-I errors with

$$
A_{0}=100 ; G_{0}=80 ; T=10 ; \mu=0.06 ; g=0.02 ; \kappa=0.01 / \sigma ; \phi=0.02 \text {. }
$$

It says that the probability that a $Q^{\theta}$-regulator claims default and liquidation coincides with the probability that under $P$ that $A_{t}^{-\theta}$ (c.f. Equation (4)) hits the barrier before maturity. Therefore, the event of false liquidation can be captured mathematically as follows:

$$
\left\{\tau^{-\theta} \leq T \text { and } \tau>T\right\}
$$

The probability for the occurrence of event (17) can be solved by using the similar techniques as in Proposition 3.1.

This can be analogously considered as Type-I error in statistics "the null hypothesis is correct but still be rejected". As an illustrative example, Table 4 lists the probability of type I error under the the standard immediate default and liquidation framework for the ambiguity-averse regulator. For an ambiguity-averse regulator (worst scenario $\theta=\kappa$ ), the false-liquidation event is equivalent to

$$
\left\{\tau^{-\kappa} \leq T \text { and } \tau>T\right\}=\left\{\inf _{t \leq T}\left\{\hat{\mu}^{* *} t+\sigma W_{t}\right\}<D_{0} \text { and } \inf _{t \leq T}\left\{m t+\sigma W_{t}\right\}>D_{0}\right\}
$$

The more risky the insurer's assets are, the more likely the Type I error occurs. Through this, the standard regulation rule turns out to be suboptimal with the time going on. This ex-post sub-optimality fact has been recognized in the literature. Bernard and Chen [2008] point out that an ex-ante optimal regulation level ceases to be optimal when the insurance company follows a risk management strategy with a non-constant volatility. In contrast, our work focuses on the information lackage/ambiguity issue. In order to mitigate the effect of ambiguity and the possible mistaken liquidation resulted from ambiguity, we illustrate in this section a new regulation rule by introducing an auditing process, which leads to a different formulation between the default and liquidation event.

\subsection{Regulation Design}

In Section 2.2.1, when determining the optimal regulation levels, the $Q^{\theta}$-regulators indeed assumes that a premature liquidation is caused when according to their beliefs $A_{t}$ hits the barrier (or under $P, A_{t}^{-\theta}$ hits the barrier). However, in reality, the regulator should not declare liquidation of the insurance company immediately, when $A_{t}^{-\theta}$ hits the barrier but the real asset value at that time $A_{t}$ is still rather high under the real world measure. This 
subsection aims to design a realistic default and liquidation mechanism where liquidation becomes in effect, only when the real assets value $A_{t}$ (instead of $A_{t}^{-\theta}$ ) under $P$ is below the barrier level. This differs from Grosen and Jørgensen [2002] and Bernard and Chen [2008]. Please note the following new default and liquidation mechanism is designed to solve the false liquidation problem. It just illustrates one possible way to better incorporate ambiguity in the default and liquidation design and can be replenished by adding some other realistic perspectives, e.g. by incorporating bankruptcy costs in the model and/or allowing the firm to be reorganized after premature liquidation.

A regulatory auditing process against the insurer's firm value $A_{t}$ is initiated whenever the monitored proxy hits the deterministic time-dependent barrier $B_{t}(\eta, \phi)$ over the monitoring times. In reality, the regulators either cannot carry out continuous monitoring or auditing or are not willing to do so due to high monitoring and/or auditing costs 6 . Therefore, in what follows, we suppose that the regulator monitors $A_{t}$ at a set of selected equidistant time points on $(0, T)$ :

$$
\mathcal{T}=\left\{t_{1}, t_{2}, \ldots, t_{N-1}\right\}, t_{j}=\frac{j T}{N}, j=1, \ldots, N-1,
$$

with $t_{N}=T$. Both proxy monitoring and regulatory auditing occur only at the time points containing in $\mathcal{T} \cup\{0, T\}$.

Suppose that it holds that a regulator with $Q^{\theta}$-beliefs observe that $A_{\bar{t}} \leq B_{\bar{t}}(\eta, \phi)$ for the first time at some particular $\bar{t} \in \mathcal{T}$. Then, the auditing process is immediately in effect and there are two possible auditing outcomes at $\bar{t}$ (under the real world measure $P$ ):

(a) $A_{\bar{t}} \leq B_{\bar{t}}(\eta, \phi)$

(b) and $A_{\bar{t}}>B_{\bar{t}}(\eta, \phi)$.

We assume that at time $\bar{t}$ the regulator resets his beliefs to the real world measure, i.e. $A_{\bar{t}+}^{-\theta}=A_{\bar{t}}$. When (1) occurs, the insurer is liquidated immediately as the insurer's true firm value has hit some "dangerous" level from the regulator's viewpoint. An immediate liquidation is therefore called for to protect the policyholder from further loss. Under scenario (2), the liquidation is not to be carried out since the insurer's prevailing true value is above the auditing level set by the regulator. Upon updating the insurer's proxy, the auditing process is stopped and the regulator resumes its usual monitoring process against the proxy. Since we have assumed that the regulators' confidence over probability measures is supposed to be not improved over time. It indicates that the regulators do not learn about the distribution of $\theta$ at time $\bar{t}$ and do not reduce the set $\mathcal{P}^{\Theta}$ over time. Note that

\footnotetext{
${ }^{6}$ Cost of enforcement includes both the necessary public funding (e.g. salaries of bureaucrats and judges, paperwork, investigations, etc.) and the compliance costs borne by audit firms.
} 
under this case the auditing process may be initiated again at a later time depending on the future evolution of the proxy. Though the regulator has full access to all historical data of $A_{t}$ for $t<\bar{t}$ at time $\bar{t}$, we have assumed that the regulator has to "forgive" and "forget" even if the regulator finds $A_{t}<B_{t}(\eta, \phi)$ for some $t<\bar{t}$ under (2). This assumption is not completely unreasonable since only the prevailing value is relevant to the insurer's insolvency at the current moment, but not its past values. Let $\tau_{L}$ denote the liquidation time of the insurer. The specification above allows us to write

$$
\tau_{L}=\inf \left\{t \in \mathcal{T} \cup\{0, T\} \mid A_{t}^{-\theta} \leq B_{t}(\eta, \phi), A_{t} \leq B_{t}(\eta, \phi)\right\}
$$

Under this setup, the auditing triggering event is not equivalent to the liquidation event. The auditing process initiated only allows the regulator to gain access to the true historical evolution of the insurer's firm value $A_{t}$.

To help understand the model specification described above, we display in Figure 1 four simulated scenarios for $A_{t}^{-\theta}$ and $A_{t}$. The black line in the figure represents the barrier $B_{t}(\eta, \phi)$, and the blue and red curves are the simulated paths of $A_{t}$ and $A_{t}^{-\theta}$, respectively. According to the regulation design, the simulated paths of $A_{t}^{-\theta}$ and $A_{t}$ on Panel 1, 2 and 3 do not lead to liquidation, although the insurer's firm value sometimes falls below the liquidation barrier. In the case of Panel 2 and 3, even no auditing takes place throughout the term of the contract, as the levels of the proxy $A_{t}^{-\theta}$ stay always above the auditing triggering barrier. On Panel 3, the real evolution of the firm's asset is for all the time above the barrier level; while the insurer on Panel 2 escapes from the potential liquidation, because $A_{t}$ does hit the barrier once. The fact $A_{t}^{-\theta}$ stays all the time above the barrier under this scenario causes no auditing. On panel 1, the auditing has been triggered before the maturity date. However, the real firm's value is still above the barrier at the auditing time. Hence, no liquidation occurs at this time. Finally on Panel 4, before auditing is implemented, the firm's asset has breached the barrier. Nevertheless, the firm cannot escape from a premature liquidation and the liquidation time is indeed identified when both $A^{-\theta}$ and $A$ simultaneously fall below the barrier for the first time. Therefore, the new default and liquidation mechanism avoids implausible liquidation (like Panel 1) enforced under the classical default and liquidation framework in which the regulator enforces liquidation according to their beliefs $Q^{\theta}$ (Technically speaking, it is equivalent to declare liquidation based on the evolution of $A_{t}^{-\theta}$ only.)

Again, we are interested in risk measures like liquidation probability. Unfortunately, it is difficult (if not impossible) to compute the the risk measure analytically under our model, unless $N$ is small. The main difficulty arises from the fact that the auditing process can be triggered at multiple times before maturity. Analytical computation requires us to keep track of the sample paths where there exist multiple auditing triggering times. Given 

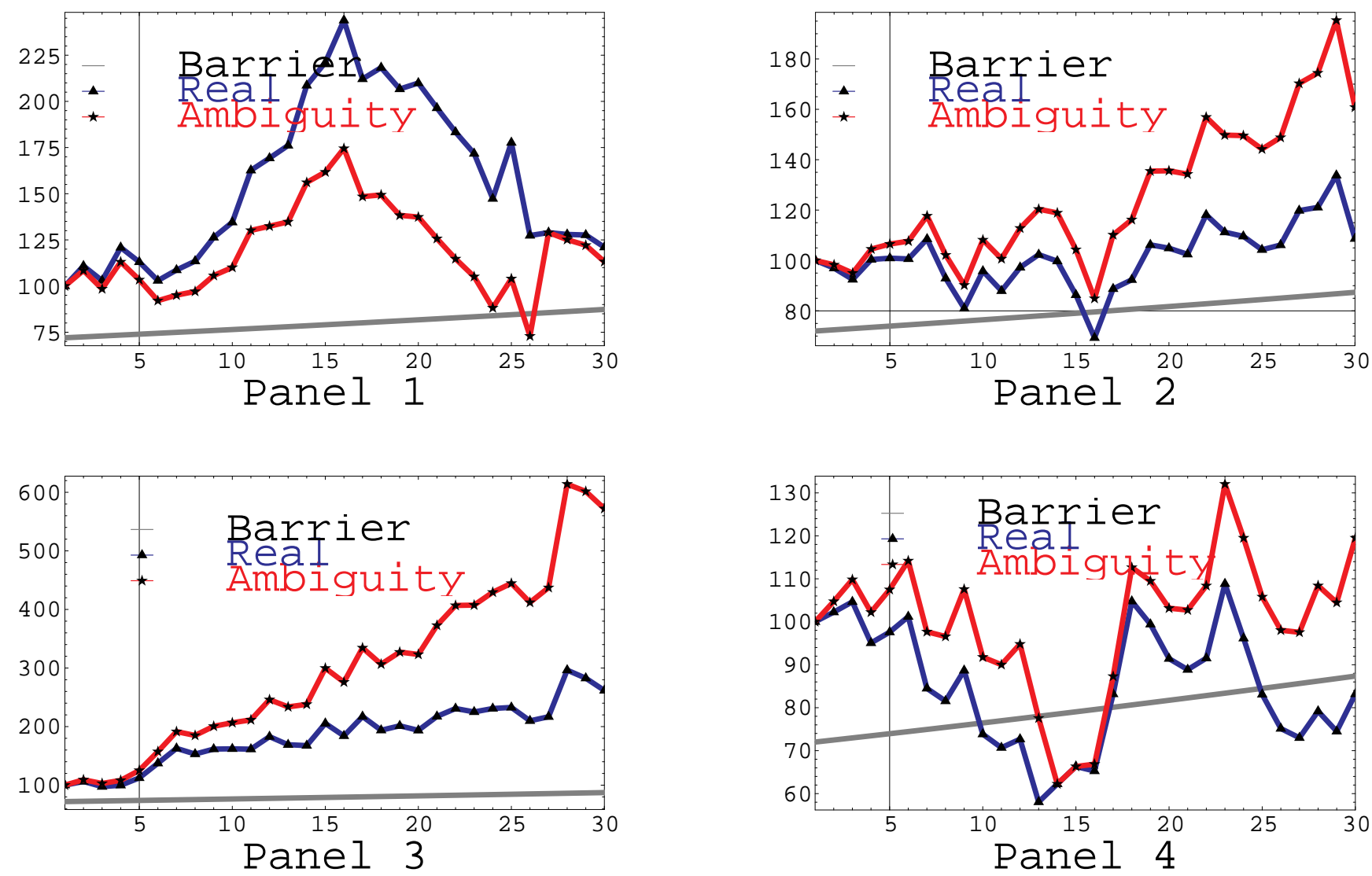

Figure 1: $\quad$ Simulated scenarios for $A_{t}^{-\theta}$ (red) and $A_{t}$ (blue): $A_{0}=100$, $G_{0}=80, \mu=5 \%, \sigma=20 \%, \eta=80 \%, \phi=2 \%, T=10, N=30, \kappa=0.01 / \sigma$. 
the long term nature of the participating contract, $N$ is usually large enough to prevent us from characterizing such sample paths in a tractable way. Fortunately, the discrete monitoring setup allows us to construct simple simulation algorithms for computing the liquidation probability. We therefore resort to Monte Carlo simulation as the main tool for our analysis in the remainder of this paper.

\subsection{Numerical result}

We illustrate some numerical results concerning the liquidation probability under the realistic model setup we propose. Throughout the simulation, a sample size of 100, 000 is used.

The analysis is firstly carried out to investigate the impacts of the volatility $\sigma$ under different debt equity ratio $(\mathrm{D} / \mathrm{E})$ and Table 5 provides the corresponding values. With $A_{0}=100$, assuming a D/E of 4 and 1 is equivalent to setting $G_{0}$ to 80 and 50, respectively. From the table, it is clear that the $\mathrm{D} / \mathrm{E}$ has a great impact on the liquidation probability. For example, for $\sigma=0.16$ and $\kappa=0.01 / \sigma$, default happens to the firm with a probability of $14.09 \%$ when $D / E=4$ (under the regulation of an ambiguity-averse regulator), whereas the probability reduces to $1.39 \%$ when $D / E=1$. This suggests that the insurer has a high probability to be liquidated if its initial equity contribution is too low compared to its initial debt. It interprets exactly the ambiguity equity introduced in Section 3.

As expected, the liquidation probabilities are increasing in $\sigma$. For $\sigma=0.1$, quite low liquidation probability results. However, a lower liquidation probability does not necessarily mean that the policyholder is better protected. For example, the regulator can always achieve a lower liquidation probability by reducing the magnitude of $\eta$ or $\phi$. By doing so, however, the amount of payment received by the policyholder in the event of liquidation is expected to be lower. Hence, the policyholder is less protected from this viewpoint. In practice, the bankruptcy of a large-sized insurer can leave many households in financial distress which imply a large amount of settlement, adminstration, and legal costs born by the regulator. Hence, it is nevertheless of great importance for the regulator to ensure that the insurer has a reasonably low shortfall probability. If one takes the insurer' standpoint, then a lower shortfall probability, of course, is always advantageous.

$\kappa$-value determines the attitude of the regulator towards ambiguity. According to the analysis of Section 3, ambiguity-averse regulators act following the worst-scenario rule, whereas ambiguity-friendly regulators act extremely optimistically. The more ambiguityaverse the regulator is, the higher default probability turns out. In contrast, the more ambiguity-friendly the regulator is, the small liquidation probability results. An ambiguityaverse regulator enforces default and liquidation as if he uses an underestimated proxy for 
monitoring and auditing, which leads to more frequent auditing processes. Consequently, it is more likely that the company is found out bankrupt. Certainly, a reversed reasoning shall hold for the ambiguity-friendly regulators. An ambiguity-neutral regulator behaves moderately and causes a shortfall probability inbetween.

\begin{tabular}{|c|c|c|c|c|c|c|c|}
\hline & \multirow[b]{2}{*}{$\mathrm{D} / \mathrm{E}$} & \multicolumn{6}{|c|}{$\sigma$} \\
\hline & & 0.10 & 0.12 & 0.14 & 0.16 & 0.18 & 0.20 \\
\hline \multirow{2}{*}{$\begin{array}{c}\text { LP (Amb.-friendly) } \\
\quad(\kappa=0.01 / \sigma)\end{array}$} & 4 & 0.0171 & 0.0472 & 0.0864 & 0.1409 & 0.1848 & 0.237 \\
\hline & 1 & 0.0001 & 0.001 & 0.0063 & 0.0139 & 0.0304 & 0.0487 \\
\hline \multirow{2}{*}{$\begin{array}{c}\text { LP (Amb.-averse) } \\
\quad(\kappa=0.01 / \sigma)\end{array}$} & 4 & 0.0308 & 0.0698 & 0.1204 & 0.1724 & 0.2388 & 0.2844 \\
\hline & 1 & 0.0005 & 0.0021 & 0.008 & 0.0203 & 0.0401 & 0.0719 \\
\hline \multirow{2}{*}{$\begin{array}{c}\text { LP (Amb.-friendly) } \\
\quad(\kappa=0.02 / \sigma)\end{array}$} & 4 & 0.0082 & 0.0323 & 0.0688 & 0.1134 & 0.1668 & 0.2084 \\
\hline & 1 & 0.0001 & 0.0011 & 0.0037 & 0.0098 & 0.0238 & 0.0424 \\
\hline \multirow{2}{*}{$\begin{array}{c}\text { LP (Amb.-averse) } \\
\quad(\kappa=0.02 / \sigma)\end{array}$} & 4 & 0.0346 & 0.0804 & 0.1304 & 0.1881 & 0.2494 & 0.3003 \\
\hline & 1 & 0.0002 & 0.0039 & 0.0112 & 0.0244 & 0.0456 & 0.0665 \\
\hline \multirow{2}{*}{$\begin{array}{c}\text { LP (Amb.-friendly) } \\
\quad(\kappa=0.05 / \sigma)\end{array}$} & 4 & 0.0006 & 0.0079 & 0.0239 & 0.0516 & 0.0825 & 0.133 \\
\hline & 1 & 0.0001 & 0.0002 & 0.0004 & 0.0032 & 0.0078 & 0.0184 \\
\hline \multirow{2}{*}{$\begin{array}{c}\text { LP (Amb.-averse) } \\
(\kappa=0.05 / \sigma)\end{array}$} & 4 & 0.0400 & 0.0988 & 0.1492 & 0.2194 & 0.2887 & 0.3397 \\
\hline & 1 & 0.0003 & 0.0033 & 0.0112 & 0.0259 & 0.0514 & 0.0853 \\
\hline
\end{tabular}

Table 5: Liquidation probabilities (LP) for different $\sigma$ values with parameters: $A_{0}=100 ; \eta=0.8 ; \mu=0.05 ; r=0.03 ; T=10 ; N=10 ; g=0.02 ; \phi=0.03$.

\section{Conclusion}

This paper investigates the substantial effect of ambiguity on insurance regulator's optimal regulation decisions. In particular, ambiguity in this paper refers to the imperfect information that insurance regulators hold about the insurance firms' future asset evolution, In this sense, our work stresses the importance of strengthening the transparency of the insurance undertaking. We calculate ambiguity equity for an ambiguity-averse regulator which is a similar concept as "ambiguity premium". Both theoretical and numerical result shows that higher equity is required just because of ambiguity in order to reach the desired default probability constraint. Furthermore, the paper demonstrates that an immediate bankruptcy policy falsely declares liquidation with a positive probability and therefore illustrates an auditory regulation rule to avoid the false declaration. This policy distinguishes the default and liquidation event, and takes an audit process before declaring backruptcy. It should be noted that our newly-developed auditory regulation rule is still 
not perfectly realistic. For instance, one of crucial factor to consider in this model is the benefit and cost of the auditing process. This is nevertheless ignored in the current work, which focuses on fixing the problem of immediate bankruptcy regulation under ambiguity. Further extension can be profit-cost analysis and also the learning process of regulators. 


\section{References}

Bacinello, A.R. (2001): "Fair Pricing of Life Insurance Participating Policies With a Minimum Interest Rate Guaranteed," ASTIN Bulletin, 31(2), 275-297.

Ballotta, L. (2005): "A Lévy Process-Based Framework for the Fair Valuation of Participating Life Insurance Contracts," Insurance: Mathematics and Economics, 37(2), 173-196.

Bernard, C., And Chen, A. (2008): "On the Regulator-Insurer-Interaction in a Structural Model," IIPR Insurance and Pension Reports, 2008-01.

Bernard, C., Le Courtois, O., and Quittard-Pinon, F. (2005): "Market Value of Life Insurance Contracts under Stochastic Interest Rates and Default Risk," Insurance: Mathematics and Economics, 36(3), 499-516.

(2006): "Development and Pricing of a New Participating Contract," North American Actuarial Journal, 10(4), 179-195.

Black, F., And Scholes, M. (1973): "The Pricing of Options and Corporate Liabilities," Journal of Political Economy, 81, 637-654.

Briys, E., and de Varenne, F. (1994): "Life Insurance in a Contingent Claim Framework: Pricing and Regulatory Implications," Geneva Papers on Risk and Insurance Theory, 19(1), 53-72.

(1997): "On The Risk of Life Insurance Liabilities: Debunking Some Common Pitfalls," Journal of Risk and Insurance, 64(4), 673-694.

(2001): Insurance from Underwriting to Derivatives. Wiley Finance.

Chen, A., And Suchanecki, M. (2007): "Default Risk, Bankruptcy Procedures and the Market Value of Life Insurance Liabilities," Insurance: Mathematics and Economics, 40(2), 231-255.

Chen, Z., and Epstein, L. (2002): "Ambiguity, Risk, and Asset Returns in Continuous Time," Econometrica, 70(4), 1403-1443.

EllsberG, D. (1961): "Risk Ambiguity and the Salvage Axioms," Quarterly Journal of Economics, 75(4), 643-669.

Grosen, A., And Jørgensen, P.L.. (2000): "Fair Valuation of Life Insurance Liabilities: The Impact of Interest Rate Guarantees, Surrender Options, and Bonus Policies," Insurance: Mathematics and Economics, 26(1), 37-57. 
(2002): "Life Insurance Liabilities at Market Value: An Analysis of Insolvency Risk, Bonus Policy, and Regulatory Intervention Rules in a Barrier Option Framework," Journal of Risk and Insurance, 69(1), 63-91.

Karatzas, I., and Shreve, S.E. (1991): "Brownian Motion and Stochastic Calculus," Springer-Verlag, New York.

Knight, F. (1921): Risk, Uncertainty, and Profit. Houghton Mifflin, Boston.

Merton, R.C. (1974): "On the Pricing of Corporate Debt: the Risk Structure of Interest Rates," Journal of finance, 29, 449-470.

(1989): "On the Application of the Continuous-Time Theory of Finance to Financial Intermediation and Insurance," Geneva Papers on Risk and Insurance Theory, $14(52), 225-261$.

Nishimura, K., and Ozaki, H. (2004): "Irreversible Investment and Knightian Uncertainty," Discussion paper, working paper.

Schmeidler, D. (1989): "Subjective Probability and Expected Utility without Additivity," Econometrica, 57(3), 571-587.

Schmeidler, D., And GilboA, I. (1989): "Maxmin Expected Utility with Nonunique Prior," Journal of Mathematical Economics, 18, 141-153.

Schulte, G. (1991): The Fall of First Executive: The House That Fred Carr Built. Harpercollins. 